\title{
Síndrome climatérica e fatores associados
}

\author{
Climacteric syndrome and associated factors \\ Síndrome climatérico y factores asociados
}

Paula Carolina Santos Oliveira das Chagas ${ }^{1 *}$, Janny Claudia Pereira Ruas ${ }^{1}$, Juliana Ferreira Vieira dos Santos ${ }^{1}$, Talita Costa Aquino Pereira ${ }^{1}$, Ester Lisboa da Silva ${ }^{1}$, Gilson Paulo Santos Nascimento', Cristiano Leonardo de Oliveira Dias', Claudia Danyella Alves Leão Ribeiro'1.

\section{RESUMO}

Objetivo: Avaliar a sintomatologia climatérica e os fatores associados. Métodos: Estudo descritivo, analítico, transversal, quantitativo, realizada em Pirapora, Minas Gerais, com 324 mulheres, de 40 a 65 anos. Utilizouse um questionário socioeconômico e clínico, a escala de Cervantes, o Índice da Função Sexual FemininaFSFI e o Índice Menopausal de Blatt-Kupperman IMBK. Tabulou-se os dados no SPSS 19.0 (Statistical Package Social Science). Resultados: A média de idade foi 51,73 anos, maioria parda $(72,0 \%)$, casada $(49,8 \%)$, com renda per capta de até 999,00 reais $(62,4 \%)$ e ensino médio incompleto/ completo (40\%). 0 percentual de risco para disfunção sexual foi $67,6 \%$. A sintomatologia climatérica mostrou-se leve em $69,4 \%$, e moderada em $30,6 \%$, e as mulheres com sintomatologia moderada tiveram escore de qualidade de vida pior do que as que apresentaram sintomatologia leve. A população avaliada teve um bom estado de saúde geral, apresentando a média de escore de 63,32. Conclusão: Observou-se que a síndrome climatérica interfere de forma negativa na vida das mulheres, pois quanto maior o impacto dos sintomas climatéricos menor é a qualidade de vida nas mulheres entrevistadas.

Palavras-chave: Climatério, Menopausa, Qualidade de vida.

\section{ABSTRACT}

Objective: To evaluate climacteric symptoms and associated factors. Methods: It is about descriptive, analytical, cross-sectional, quantitative study, conducted in Pirapora, Minas Gerais, with 324 women, aged 40 to 65 years. A socioeconomic and clinical questionnaire, the Cervantes scale, the Female Sexual Function Index - FSFI and the Blatt-Kupperman IMBK Menopausal Index, were used. Data were tabulated in SPSS 19.0 (Statistical Package Social Science). Results: The average age was 51.73 years old, brown majority (72.0\%), married (49.8\%), with per capita income of up to 999.00 real $(62.4 \%)$ and incomplete high school / complete $(40 \%)$. The risk percentage for sexual dysfunction was $67.6 \%$. Climacteric symptoms were mild in $69.4 \%$, and moderate in $30.6 \%$, and women with moderate symptoms had a worse quality of life score than those with mild symptoms. The evaluated population had a good general health status, with a mean score of 63.32. Conclusion: It was observed that the climacteric syndrome negatively affects the lives of women, because the greater the impact of climacteric symptoms, the lower the quality of life in the women interviewed.

Key words: Climacteric, Menopause, Quality of life.

\section{RESUMEN}

Objetivo: Evaluar los síntomas climatéricos y los factores asociados. Métodos: Estudio descriptivo, analítico, transversal, cuantitativo, realizado en Pirapora, Minas Gerais, con 324 mujeres, de 40 a 65 años. Se utilizó

1Universidade Estadual de Montes Claros (Unimontes). Pirapora - MG.

*E-mail: paula87pirapora@yahoo.com.br

SUBMETIDO EM: 4/2020

ACEITO EM: 4/2020

PUBLICADO EM: 7/2020 
un cuestionario socioeconómico y clínico, la escala de Cervantes, el índice de función sexual femenina - FSFI y el índice de menopausia IMBK Blatt-Kupperman. Los datos se tabularon en SPSS 19.0 (Statistical Package Social Science). Resultados: La edad promedio fue de 51.73 años, mayoría marrón (72.0\%), casada (49.8\%), con un ingreso per cápita de hasta 999.00 reales $(62.4 \%)$ y escuela secundaria incompleta / completo $(40 \%)$. El porcentaje de riesgo de disfunción sexual fue del $67,6 \%$. Los síntomas climáticos fueron leves en el $69,4 \%$ y moderados en el 30,6\%, y las mujeres con síntomas moderados tuvieron una peor puntuación de calidad de vida que aquellas con síntomas leves. La población evaluada tenía un buen estado de salud general, con una puntuación media de 63,32. Conclusión: se observó que el síndrome climatérico afecta negativamente la vida de las mujeres, porque cuanto mayor es el impacto de los síntomas climatéricos, menor es la calidad de vida de las mujeres entrevistadas.

Palabras clave: Climaterio, Menopausia, Calidad de Vida.

\section{INTRODUÇÃO}

O climatério é uma etapa fisiológica do ciclo da vida da mulher, que corresponde à transição entre a fase reprodutiva e não reprodutiva. Geralmente, a fase climatérica ocorre entre os 40 e 65 anos. A menopausa corresponde à interrupção definitiva da menstruação e o diagnóstico clínico é feito após 12 meses consecutivos de amenorreia, ocorrendo, habitualmente, entre os 48 e 50 anos de idade (BRASIL, 2016).

A causa da ocorrência do climatério deve-se ao fato dos ovários sofrerem uma diminuição gradativa que vai de 8 a 9 centímetros na fase reprodutiva para 2 a 3 centímetros na pós-menopausa. A função ovariana sofre alteração em decorrência da diminuição da produção estrogênica e aumento das gonadotrofinas hipofisárias causando o que se denomina de hipogonadismo hipergonadotrófico (ROCHA ASG, 2017).

O hipoestrogenismo pode ser um fator determinante da sintomatologia ou síndrome climatérica, que se apresentam na maioria das vezes com manifestações neurogênicas. Os sintomas típicos são fogachos, sudorese, calafrios, palpitações, cefaleia, parestesias, insônia, fadiga, além de irritabilidade, alterações dos estados psicológicos e diminuição da resistência no corpo associada à baixa calcificação nos ossos, o que acaba comprometendo o sistema musculoesquelético (DEDICAÇÃO AC, 2012).

Além dos sintomas frequentes da síndrome climatérica, outro agravante à saúde das mulheres nesse período é o ganho de gordura corporal podendo levá-las à obesidade. Isso ocorre por múltiplos fatores como a redução do metabolismo relacionada ao hipoestrogenismo, mudanças no estilo de vida, e o consumo de alimentos mais calóricos. A obesidade é caracterizada como uma disfunção metabólica, e estima-se que no Brasil $64,9 \%$ das mulheres são obesas ou estão com sobrepeso, ademais, percebe-se maior prevalência na faixa etária entre 55 a 64 anos, coincidindo com a fase climatérica (GONÇALVES JTT, et al., 2016).

O Ministério da Saúde constatou que em 2011, 55,9\% da população feminina entre 45 e 54 anos estava obesa, enquanto a de 55 a 65 anos possuía uma taxa de 60,9\%. O sobrepeso e a obesidade tornam-se um problema de saúde pública a ser enfrentado, uma vez que elevam o desenvolvimento de doenças crônicas nas mulheres após os 50 anos (BRASIL, 2011; GONÇALVES JTT, et al., 2016).

Apesar de a sintomatologia clínica ser o alvo das ações dos profissionais às mulheres climatéricas, é necessário atentar também às alterações no âmbito familiar e social que igualmente são considerados fatores relevantes para a avaliação do enfrentamento dessa mulher perante as mudanças ocorridas nessa fase (FREITAS ER, et al., 2016).

Dentre os fatores sociais que podem afetar a vida das mulheres climatéricas estão: a saída dos filhos de casa, o cuidado com os pais idosos, as mudanças fisiológicas corporais, a perda de parentes e amigos, uma nova perspectiva sobre a sexualidade e o relacionamento, tudo isso são fatores que alteram o cotidiano dessa mulher e, consequentemente, podem prejudicar a sua qualidade de vida (QV) (FREITAS ER, et al., 2016). A definição de Qualidade de vida (QV) é tida como a percepção do indivíduo sobre a sua posição na vida, no contexto da cultura e dos sistemas de valores nos quais ele vive, e em relação aos seus objetivos, 
expectativas, padrões e preocupações (FREITAS ER e BARBOSA AJG, 2015). Dessa forma, a QV é construída numa perspectiva holística, que sofre influência do estado psíquico, biológico e social, sendo ainda variável de acordo com o contexto de vida vivido pelos sujeitos (FREITAS ER, et al., 2016).

No que tange a assistência à mulher climatérica no âmbito de sua sexualidade a abordagem vai além das questões fisiológicas, é importante observar os aspectos socioculturais, as condições de vida e a percepção das mulheres diante do fenômeno do climatério (SANTOS RCF, et al., 2017).

Além desses fatores extrínsecos, existem também os intrínsecos que contribuem para o abandono das práticas sexuais nesse período. Dentre as causas intrínsecas destaca-se o déficit de estrogênio que torna epitélio do trato genital menos espesso e frágil. Na região vulvar, ocorre déficit na secreção das glândulas sudoríparas, sebáceas e atrofia das glândulas de Bartholin. Sendo assim, com essas alterações fisiológicas ocorre uma menor capacidade de lubrificação diante do estímulo sexual levando à dispareunia (MARTINS M, et al., 2015).

A disfunção sexual feminina (DSF) pode ser classificada em: transtornos do desejo sexual, desejo sexual hipoativo (DSH), aversão sexual, transtorno de excitação, transtorno do orgasmo feminino (atraso ou ausência persistente ou recorrente de orgasmo, após uma fase normal de excitação sexual), transtornos sexuais dolorosos (dispareunia ou vaginismo), disfunção sexual devido a uma condição médica e disfunção sexual induzida por substâncias (SANTOS SJ, et al., 2016).

Partindo da importância do assunto para o âmbito de saúde pública é relevante que a orientação acerca das modificações climatéricas seja um instrumento para promoção de saúde. Portanto é imprescindível que as mulheres que apresentam queixas relacionadas ao climatério sejam devidamente preparadas e encorajadas para que os impactos dessas modificações sejam enfrentados de forma menos impactante (ALVES ERP, et al., 2015).

É essencial que os profissionais de saúde entendam e ajudem às mulheres a compreenderem que envelhecer é um processo natural, e o cuidado voltado para esse público deve ser baseado em princípios éticos aliados a competências relacionais, aconselhamento, orientações e educação para a saúde e a qualidade de vida (BRASIL, 2016). Nesse contexto, este estudo tem como objetivo avaliar os sintomas das mulheres na fase climatérica e os fatores associados.

\section{MÉTODOS}

Trata-se de um estudo descritivo, analítico, transversal de caráter quantitativo. A pesquisa foi realizada no município de Pirapora, Minas Gerais, em quatro equipes de Estratégia de Saúde da Família: Cícero Passos, Joseilson Fonseca, Antônio Senra e Santo Antônio. Participaram do estudo mulheres na fase climatérica cadastradas nas referidas equipes de saúde da família. A população total do estudo foi de 2.045 mulheres, de acordo com o cadastro no sistema VIVVER, que é um sistema de informações do município integrado ao sistema e-sus.

A seleção da amostra foi realizada com base na técnica da amostragem aleatória simples. Foi feito uma lista numerada de todas as participantes e realizado o sorteio. O tamanho da amostra foi calculado visando estimar os parâmetros populacionais com prevalência de 50\%, nível de confiança de $95 \%$, e precisão de $5 \%$, estimando a participação de 324 mulheres. Foram utilizados como critério de inclusão mulheres em fase climatérica, de 40 a 65 anos cadastradas nas equipes de Saúde da Família selecionadas.

Os critérios de exclusão foram mulheres que fizeram histerectomia total, mulheres em Terapia de Reposição Hormonal (TRH), mulheres que não tinham capacidade cognitiva para responder aos questionários ou que fossem acamadas e as mulheres analfabetas (em decorrência da autoaplicação do questionário). Para a coleta de dados foi utilizado um questionário elaborado pelas autoras, que contempla condições socioeconômicas, clínicas, perfil antropométrico (peso, altura, Índice de Massa Corporal (IMC) e circunferência abdominal). O IMC foi calculado através da fórmula IMC = peso (Kg) / (altura $x$ altura) ( $\mathrm{m}$ ), após obtenção do resultado classificamos em: baixo peso $\left(<18,5 \mathrm{~kg} / \mathrm{m}^{2}\right)$; eutrófico $\left(18,5-24,9 \mathrm{~kg} / \mathrm{m}^{2}\right)$; sobrepeso 
$\left(25-29,9 \mathrm{~kg} / \mathrm{m}^{2}\right)$ e obesidade ( $\geq 30 \mathrm{~kg} / \mathrm{m}^{2}$ ) (GONÇALVES JTT, et al., 2016). Considerou-se risco aumentado para doenças cardiovasculares mulheres com circunferência abdominal maior do que 80 centímetros (FREITAS RF, et al., 2014).

Para a avaliação da qualidade de vida foi utilizado a escala de Cervantes. A escala de Cervantes (EC) foi criada na Espanha, em 2002. Essa escala é composta por 31 questões e já foi validada no Brasil por Lima JEM (2009). Ela tem a capacidade de mensurar a QV da mulher no período pré e pós-menopausa. A vantagem desse instrumento é que ele inter-relaciona outros fatores com a sintomatologia climatérica, tendo uma avaliação mais realista da QV (LIMA JEM, 2009).

Para a avaliação da sexualidade foi utilizado um instrumento denominado de Female Sexual Function Index /Índice da Função Sexual Feminina-FSFI. O FSFI foi validado no Brasil em 2008 por Thiel e colaboradores (THIEL RRC, et al., 2008). Ele é específico e multidimensional à resposta sexual feminina, é composto por 19 questões, que informam sobre cinco domínios da resposta sexual: desejo e estímulo subjetivo, lubrificação, orgasmo, satisfação e dor ou desconforto.

As pontuações individuais são obtidas pela soma dos itens que compreendem cada domínio (escore simples), que são multiplicadas pelo fator desse domínio e fornecem o escore ponderado.

A pontuação final (escore total: mínimo de 2 e máximo de 36) é obtida pela soma dos escores ponderados de todos os domínios (SANTOS SJ, et al., 2016). Para avaliação da sintomatologia utilizou-se o Índice Menopausal de Blatt-Kupperman-IMBK. Apesar desse instrumento não ser validado no Brasil, ele é amplamente utilizado, uma vez que através dele é possível diagnosticar as pacientes portadoras de síndrome climatérica e identificar a intensidade da sintomatologia em leve, moderada e intensa (SILVEIRA IL, et al., 2007).

O IMBK possui 11 sintomas pontuados, as respostas para cada sintoma investigado seguem a seguinte escala de escores: 0 (ausência de sintomas); 1 (sintomas leves); 2 (sintomas moderados) e 3 (sintomas intensos). Para o cálculo do escore total, os sintomas apresentam pesos variados, nos quais as ondas de calor (fogachos) assumem maior relevância (peso 4), parestesia, insônia e nervosismo um valor intermediário (peso 2) e os demais sintomas, como depressão, vertigens, fadiga, artralgia/ mialgia, cefaleia, palpitação e zumbido, peso 1.

Multiplicando a intensidade do sintoma pelo fator de conversão e, em seguida, fazendo a soma dos resultados, alcança-se uma pontuação capaz de classificar a síndrome climatérica em leve, moderada e intensa. A síndrome climatérica de intensidade leve é a de pontuação até 19, moderada entre 20 e 35 e intensa maior que 35 (POLISSENI AF, et al., 2009). Os dados foram tabulados por meio de software estatístico SPSS (Statistical Package Social Science), versão 19.0. Foi realizada uma análise univariada de prevalência por frequência absoluta $(n)$ e relativa (\%). Também foram calculadas as medidas de tendência central (média, mediana, moda e desvio padrão).

Na segunda etapa, desenvolveu-se uma análise bivariada das variáveis paramétricas, através de testes estatísticos como o teste qui-quadrado de Pearson, teste t de Student e Anova 1 fator, levando em consideração intervalo de confiança (IC) de $95 \%$, erro padrão (EP) de $5 \%$ e p valor $<0,05$.

A coleta de dados ocorreu entre setembro de 2019 a fevereiro de 2020. As mulheres sorteadas foram convidadas a comparecerem na unidade de saúde para participarem de grupos, outras foram visitadas em seus domicílios pelos pesquisadores, onde foram sensibilizadas sobre a importância da pesquisa, informadas sobre a metodologia do estudo, seus objetivos e o anonimato das informações.

As que aceitaram participar da pesquisa assinaram o Termo de Consentimento Livre e Esclarecido e tiveram suas medidas aferidas. A presente pesquisa atende o que determina a Resolução o 466 de 2012, que regulamenta pesquisas envolvendo seres humanos. Foi solicitada a autorização da secretária municipal de saúde para execução da pesquisa, posteriormente, a pesquisa foi submetida para aprovação pelo Comitê de Ética em pesquisa da Universidade Estadual de Montes Claros - Unimontes, sendo que após aprovação com número de parecer 13124819.8.0000.5146, iniciou-se as coletas de dados. 


\section{RESULTADOS}

Na Tabela 1, estão apresentadas as características da amostra estudada de 324 mulheres. Os dados evidenciam que a maioria da população estudada tem entre 51-60 anos (41,4\%). A cor/raça autodeclarada mais prevalente foi a parda $72,0 \%$. Quanto ao estado civil, a maioria das entrevistadas eram casadas $(49,8 \%)$ e renda per capta de até 999,00 reais $(62,4 \%)$.

De acordo com a análise sobre o grau de escolaridade, a pesquisa revelou que $40 \%$ possuíam o ensino médio incompleto/ completo.

No que diz respeito aos cuidados com a saúde, 92,3\% negam tabagismo e $80,2 \%$ etilismo. Quanto à existência de doenças crônicas, constatou-se que $54,2 \%$ possui pelo menos uma patologia, e $62,1 \%$ fazem uso de medicação contínua.

Com relação ao risco para doenças metabólicas através da medida obtida da circunferência abdominal, verificou-se que $79,6 \%$ apresentam risco para essas doenças, uma vez que tiveram uma medida maior que 80 centímetros. Avaliando o IMC, observou-se que uma prevalência de mulheres com obesidade grau I, II e III $(40,3 \%)$.

Ao observar o escore total obtido no questionário FSFI, evidenciou-se que a maioria das mulheres participantes desse estudo apresenta algum tipo de disfunção sexual (67,6\%). A sintomatologia climatérica mostrou-se leve em $69,4 \%$ das participantes e moderada em 30,6\%, não houve sintomatologia intensa na população estudada no que se refere à somatória de todos os sintomas avaliados pelo Índice menopausal de Kupperman e Blat. 
Tabela 1 - Quantitativo e frequência das características sociodemográficas e clínicas das mulheres climatéricas estudadas. Pirapora - MG, 2020.

\begin{tabular}{|c|c|c|}
\hline Variáveis & $\mathbf{N}$ & $\%$ \\
\hline \multicolumn{3}{|c|}{ Sociodemográfico } \\
\hline \multicolumn{3}{|l|}{ Idade } \\
\hline Até 40 anos & 9 & 2,8 \\
\hline 41 a 50 anos & 132 & 40,7 \\
\hline 51 a 60 anos & 134 & 41,4 \\
\hline Acima de 60 anos & 49 & 15,1 \\
\hline \multicolumn{3}{|l|}{ Cor da pele } \\
\hline Preta & 35 & 11,3 \\
\hline Parda & 224 & 72,0 \\
\hline Branca & 49 & 15,8 \\
\hline Amarela & 3 & 1,0 \\
\hline \multicolumn{3}{|l|}{ Estado civil } \\
\hline Solteira & 99 & 31,4 \\
\hline Casada & 157 & 49,8 \\
\hline Amasiada & 38 & 12,1 \\
\hline Viúva & 21 & 6,7 \\
\hline \multicolumn{3}{|l|}{ Renda } \\
\hline Até 999 reais & 128 & 62,4 \\
\hline 1000 a 2000 reais & 50 & 24,4 \\
\hline Acima de 2000 reais & 27 & 13,2 \\
\hline \multicolumn{3}{|l|}{ Escolaridade } \\
\hline $\begin{array}{l}\text { Ensino fundamental } \\
\text { incompleto/completo }\end{array}$ & 125 & 39,7 \\
\hline Ensino médio incompleto/completo & 126 & 40,0 \\
\hline Ensino superior incompleto/completo & 64 & 20,3 \\
\hline
\end{tabular}




\section{Revista Eletrônica Acervo Saúde / Electronic Journal Collection Health | ISSN 2178-2091}

\begin{tabular}{|c|c|c|}
\hline \multicolumn{3}{|l|}{ Cuidados com a saúde } \\
\hline \multicolumn{3}{|l|}{ Tabagista } \\
\hline Não & 288 & 92,3 \\
\hline Sim & 24 & 7,7 \\
\hline \multicolumn{3}{|l|}{ Etilista } \\
\hline Não & 211 & 80,2 \\
\hline Sim & 52 & 19,8 \\
\hline \multicolumn{3}{|l|}{ Patologia(s) } \\
\hline Não & 135 & 45,8 \\
\hline Sim & 160 & 54,2 \\
\hline \multicolumn{3}{|l|}{ Uso de medicamento } \\
\hline Não & 117 & 37,9 \\
\hline Sim & 192 & 62,1 \\
\hline \multicolumn{3}{|l|}{ C. cintura } \\
\hline Sem risco para doenças metabólicas & 65 & 20,4 \\
\hline Risco para doenças metabólicas & 254 & 79,6 \\
\hline \multicolumn{3}{|l|}{ IMC } \\
\hline Abaixo do peso & 1 & 0,3 \\
\hline Peso normal & 82 & 26,0 \\
\hline Sobrepeso & 105 & 33,4 \\
\hline Obesidade & 127 & 40,3 \\
\hline \multicolumn{3}{|l|}{ Disfunção sexual } \\
\hline Sem risco para disfunção sexual & 67 & 32,4 \\
\hline Risco para disfunção sexual & 140 & 67,6 \\
\hline \multicolumn{3}{|l|}{ Kupperman } \\
\hline Leve & 163 & 69,4 \\
\hline Moderado & 72 & 30,6 \\
\hline Intenso & 0 & 0,0 \\
\hline
\end{tabular}

Fonte: Chagas PCSO, et al., 2020. 
Na Tabela 2 estão apresentados os dados obtidos através da média, mediana e desvio padrão dos aspectos sociodemográficos e clínicos das mulheres entrevistadas. Esses valores consistem em idade, renda mensal, circunferência abdominal, Índice de Massa Corporal e nível de qualidade de vida avaliado através do escore obtido na Escala de Cervantes.

Tabela 2 - Caracterização da amostra. Dados obtidos através de média, mediana e desvio padrão dos aspectos sociodemográficos e clínicos das entrevistadas. Pirapora - MG, 2020.

\begin{tabular}{lccc}
\hline \multicolumn{1}{c}{ Variáveis } & Média & Mediana & Desvio padrão \\
\hline Idade & 51,73 & 51,00 & 6,72 \\
Renda & $1.175,00$ & 998,00 & $1.160,29$ \\
C. cintura & 90,33 & 91,00 & 14,20 \\
IMC & 28,89 & 28,27 & 5,57 \\
Cervantes & 63,32 & 62,00 & 29,06 \\
\hline
\end{tabular}

Fonte: Chagas PCSO, et al., 2020.

A Tabela 3 representa os valores absolutos e o percentual de cada sintoma avaliado pelo Índice Menopausal de Kupperman e Blat separadamente, o que evidencia presença de sintomas leves, moderados e intensos quando avaliados de maneira isolada, o que é diferente da sintomatologia ou escore geral leve, moderado e intenso, que representa a somatória de todos eles.

A respeito da intensidade dos sintomas os mais citados foram: artralgia e mialgia $(33,4 \%)$, ondas de calor $(32,8 \%)$ e nervosismo $(32,5 \%)$, enquanto os sintomas mais vivenciados foram as ondas de calor (314), insônia (307) e nervosismo (305).

Tabela 3 - Distribuição e proporção das mulheres climatéricas, segundo a presença dos sintomas descritos no Índice Menopausal de Kupperman e Blat. Pirapora - MG, 2020.

\begin{tabular}{lccc}
\hline \multicolumn{1}{c}{ Variáveis } & Leve $\mathbf{n ~ ( \% )}$ & Moderado $\mathbf{~}(\%)$ & Intenso $\mathbf{~ ( \% ) ~}$ \\
\hline Ondas de calor & $115(36,6)$ & $96(30,6)$ & $103(32,8)$ \\
Parestesia & $182(63,9)$ & $66(23,2)$ & $37(13,0)$ \\
Insônia & $152(49,5)$ & $77(25,1)$ & $78(25,4)$ \\
Nervosismo & $107(35,1)$ & $99(32,5)$ & $99(32,5)$ \\
Depressão & $177(64,1)$ & $57(20,7)$ & $42(15,2)$ \\
Fadiga & $150(51,2)$ & $78(26,6)$ & $65(22,2)$ \\
Artralgia/mialgia & $128(44,6)$ & $63(22,0)$ & $96(33,4)$ \\
Cefaleia & $149(51,6)$ & $94(32,5)$ & $46(15,9)$ \\
Palpitação & $160(55,4)$ & $76(26,3)$ & $53(18,3)$ \\
Zumbindo no ouvido & $193(67,7)$ & $51(17,9)$ & $41(14,4)$ \\
\hline
\end{tabular}

Fonte: Chagas PCSO, et al., 2020.

Dentre as variáveis apresentadas na Tabela 4, verificou-se que as variáveis: renda $(p=0,034)$, patologia (s) ( $p=0,047)$, uso de medicamento ( $s)(p=0,011)$ e Índice Menopausal de Kupperman e Blat $(p=0,0001)$ foram significativas, apresentando associação com a qualidade de vida no climatério (Escala de Cervantes). 
Tabela 4 - Associação entre Escala de Cervantes e as variáveis sociodemográficas e clínicas em mulheres climatéricas. Pirapora - MG, 2020.

\begin{tabular}{|c|c|c|c|c|}
\hline Variáveis & $\mathbf{N}$ & Média & Desvio Padrão & $\mathbf{P}$ \\
\hline \multicolumn{5}{|l|}{ Idade } \\
\hline Até 40 anos & 6 & 69,00 & 38,21 & \multirow{4}{*}{0,103} \\
\hline 41 a 50 anos & 50 & 74,22 & 30,04 & \\
\hline 51 a 60 anos & 48 & 64,75 & 26,57 & \\
\hline Acima de 60 anos & 14 & 50,21 & 37,00 & \\
\hline \multicolumn{5}{|l|}{ Cor da pele } \\
\hline Preta & 15 & 60,00 & 25,40 & \multirow{3}{*}{0,160} \\
\hline Parda & 85 & 69,14 & 31,76 & \\
\hline Branca & 17 & 61,17 & 27,34 & \\
\hline \multicolumn{5}{|l|}{ Estado Civil } \\
\hline Solteira & 33 & 63,24 & 34,53 & \multirow{4}{*}{0,093} \\
\hline Casada & 63 & 68,88 & 28,39 & \\
\hline Amasiada & 14 & 78,14 & 32,32 & \\
\hline Viúva & 8 & 51,87 & 21,93 & \\
\hline \multicolumn{5}{|l|}{ Renda } \\
\hline Até 999 reais & 79 & 72,98 & 31,70 & \multirow{3}{*}{0,034} \\
\hline 1000 a 2000 reais & 29 & 57,31 & 29,43 & \\
\hline Acima de 2000 reais & 16 & 59,12 & 20,65 & \\
\hline \multicolumn{5}{|l|}{ Escolaridade } \\
\hline $\begin{array}{l}\text { Ensino fundamental } \\
\text { incompleto/completo }\end{array}$ & 46 & 68,97 & 33,33 & \multirow{3}{*}{0,170} \\
\hline $\begin{array}{c}\text { Ensino médio } \\
\text { incompleto/completo }\end{array}$ & 47 & 70,25 & 30,91 & \\
\hline $\begin{array}{c}\text { Ensino superior } \\
\text { incompleto/completo }\end{array}$ & 25 & 58,40 & 23,14 & \\
\hline \multicolumn{5}{|l|}{ IMC } \\
\hline Peso normal & 30 & 70,76 & 30,29 & \multirow{3}{*}{0,122} \\
\hline Sobrepeso & 32 & 62,40 & 30,05 & \\
\hline Obesidade & 56 & 68,14 & 31,20 & \\
\hline
\end{tabular}

REAS/EJCH | Vol.Sup.n.51 | e3536 | DOI: https://doi.org/10.25248/reas.e3536.2020 Página 9 de 13 
Revista Eletrônica Acervo Saúde / Electronic Journal Collection Health | ISSN 2178-2091

\begin{tabular}{|c|c|c|c|c|}
\hline Tabagismo & & & & \\
\hline Não & 175 & 63,057 & 29,69 & \multirow{2}{*}{0,522} \\
\hline Sim & 14 & 68,214 & 16,65 & \\
\hline \multicolumn{5}{|l|}{ Etilismo } \\
\hline Não & 127 & 62,228 & 28,864 & \multirow{2}{*}{0,227} \\
\hline Sim & 34 & 69,058 & 30,280 & \\
\hline \multicolumn{5}{|l|}{ Patologia(s) } \\
\hline Não & 88 & 58,875 & 31,148 & \multirow{2}{*}{0,047} \\
\hline Sim & 93 & 67,526 & 26,921 & \\
\hline \multicolumn{5}{|l|}{ Uso de medicamento(s) } \\
\hline Não & 76 & 56,736 & 28,915 & \multirow{2}{*}{0,011} \\
\hline Sim & 107 & 67,831 & 28,772 & \\
\hline \multicolumn{5}{|l|}{ C. cintura } \\
\hline Sem risco para doenças & 32 & 62,687 & 24,715 & \multirow{3}{*}{0,952} \\
\hline $\begin{array}{c}\text { metabolicas } \\
\text { Risco para doenças }\end{array}$ & & & & \\
\hline $\begin{array}{l}\text { metabólicas } \\
\text { mes }\end{array}$ & 158 & 63,025 & 29,917 & \\
\hline \multicolumn{5}{|l|}{ Kupperman } \\
\hline Leve & 104 & 54,375 & 24,274 & \multirow{2}{*}{0,0001} \\
\hline Moderado & 50 & 84,54 & 24,018 & \\
\hline \multicolumn{5}{|l|}{ Disfunção sexual } \\
\hline Sem risco para disfunção & 50 & 72,060 & 27,701 & \multirow{3}{*}{0,071} \\
\hline sexual & 60 & & & \\
\hline $\begin{array}{l}\text { Risco para disfunção } \\
\text { sexual }\end{array}$ & 95 & 63,094 & 28,438 & \\
\hline
\end{tabular}




\section{DISCUSSÃO}

Após o aumento considerável na expectativa de vida das brasileiras nas últimas décadas, grande parte das de mulheres vem vivenciando o climatério por um período maior, essa mudança demográfica causa um redirecionamento das queixas dessas mulheres que podem causar um impacto em sua qualidade de vida. Assim, ao analisar o perfil sociodemográfico evidenciou-se uma idade média de 51,73 anos, sendo maioria de mulheres casadas, com ensino médio incompleto/completo, esses dados corroboram com outros estudos analisados como o de Andrade RL, et al. (2019), que encontrou respectivamente os seguintes resultados para as mesmas variáveis: 53,$5 ; 62,2 \%$ e 35,1\% (ANDRADE RL, et al., 2019). Destaca-se ainda que esse aumento da longevidade requeira uma remodelação da forma de atenção voltada a essas mulheres climatéricas no âmbito de politicas de saúde publica, uma vez que atender essa população é uma prática de integralidade à saúde da mulher.

No estudo realizado por Andrade $\mathrm{RL}$, et al. (2019), o índice de não etilistas (97,3\%) e não tabagistas $(91,9 \%)$ foram majoritários, assim como na presente pesquisa que obteve, respectivamente, $80,2 \%$ e $92.3 \%$ não etilistas e não fumantes. Ainda sobre os mesmos autores, detectou-se que, $73 \%$ das mulheres entrevistadas tinham risco elevado para doenças cardiovasculares, avaliada pela elevada circunferência abdominal $(>80 \mathrm{~cm}$ ) assim como neste estudo, o qual obteve um percentual de 79,6, o que confirma achados presentes na literatura (ANDRADE RL, et al., 2019; FREITAS RF, et al., 2015; FREITAS RF, et al., 2017).

Um dos fatores que afetam a qualidade de vida das pessoas é a obesidade, porém, no Brasil, $64,9 \%$ das pessoas do sexo feminino apresentam diagnóstico nutricional de sobrepeso e obesidade, sendo sua frequência maior na faixa etária de 55 a 64 anos (IBGE, 2010). A redução do metabolismo é a principal causa do ganho de peso no climatério/menopausa o que promove acréscimo de 250g/ano na massa corpórea (PEREIRA DCL e LIMA SMRR, 2015). Este estudo observou que, além da elevada circunferência abdominal o perfil antropométrico prevalente nas mulheres climatéricas corroboram com os estudos de maior parte dos trabalhos pesquisados que certificaram o predomínio de sobrepeso e obesidade nas mulheres neste período da vida (MELO C, et al., 2016; RIBEIRO AS, et al., 2015; GONCALVES JTT, et al., 2016; RODRIGUES RM, 2019).

Neste estudo, a intensidade dos sintomas climatéricos apresentados resultou em $69,4 \%$ das mulheres com sintomatologia climatérica leve e $30,6 \%$ moderada, sendo que a sintomatologia intensa foi ausente na população estudada. Resultados que foram observados em outros trabalhos, também constataram a prevalência da sintomatologia leve sobre a moderada e a intensa. Um estudo realizado com 80 mulheres, de 40 a 60 anos, revelou que $58,7 \%$ das mulheres tinham escore total de sintomas climatéricos leves, $31,3 \%$ moderados e 10\% intensos (MELO C, et al., 2016), outro estudo que avaliou 303 mulheres com idade entre 45 e 60 anos, apresentou condição semelhante, tendo $41,92 \%$ com sintomas leves, $35,64 \%$ moderados e 22,4\% intensos (ARAÚJO JBS, et al., 2015)

As alterações que abrangem mulheres climatéricas causadas pelo hipoestrogenismo geram sintomas que afeta a maioria delas (BESSA AD, et al., 2016). A artralgia e mialgia foram citadas como os sintomas mais intensos totalizando $33,4 \%$ das que afirmam sentir dores articulares e musculares, seguido por ondas de calor $(32,8 \%)$ e nervosismo $(32,5 \%)$. Os sintomas mais vivenciados foram ondas de calor, sendo queixa frequente de 314 mulheres no total, acompanhadas, respectivamente, por insônia (307) e nervosismo (305). Assim como nos estudos de Bessa AD, et al. (2016), Araújo JBS et al. (2015) e Alves ERP, et al. (2015), a intensidade da síndrome climatérica foi predominantemente classificada como leve $(69,4 \%)$ (BESSA AD, et al., 2016; ARAÚJO JBS, et al., 2015; ALVES ERP, et al., 2015).

A respeito desses sintomas descritos acima, esse estudo avaliou também o impacto da síndrome climatérica na qualidade de vida e função sexual das mulheres, o que apontou uma correlação significativa entre os aspectos de qualidade de vida avaliados pela escala de Cervantes e os sintomas climatéricos verificados pelo Índice Menopausal de Kuppermam e Blat. Semelhante aos trabalhos de Rocha AGS (2017) e MIRANDA JS et al. (2014), a população avaliada apresentou bom estado de saúde de modo geral, uma vez que a média do escore total obtida foi de 63,32 $( \pm 29,6)$ (ROCHA AGS, 2017; MIRANDA JS, et al., 2014).

REAS/EJCH | Vol.Sup.n.51 | e3536 | DOI: https://doi.org/10.25248/reas.e3536.2020 Página 11 de 13 
Obteve uma melhor qualidade de vida as mulheres que possuíam renda per-capta mais alta em detrimento das possuíam renda até 999,00 reais $(p=0,034)$, fato comprovado também nos estudos de outros autores que encontraram significância entre as referidas variáveis (FREITAS RF, et al., 2014; LIMA CG, et al., 2011; LORENZI D, et al., 2006).

Entende-se que o enfermeiro deve promover o cuidado às mulheres climatéricas e menopausadas baseando-se na sintomatologia relatada por cada mulher de forma que atenda a singularidade de cada uma através de ações educacionais e orientações que fortaleçam uma maior autonomia e autocuidado nessa etapa da vida.

Existe uma escassez de dados epidemiológicos no Brasil sobre síndrome climatérica, portanto, acreditase que um estudo de base populacional sobre esta temática é de grande relevância para um melhor entendimento de como as mulheres enfrentam essa fase da vida e quais são os fatores que se associam a um comprometimento da qualidade de vida. Entretanto, esta pesquisa apresenta limitações como o fato do estudo ter sido aplicado em apenas quatro das dezessete Estratégias de Saúde da Família, o que não representa todo o município de Pirapora. Outra limitação foi a falta de informação sobre o estado menopausal (pré-menopausa, perimenopausa e pós-menopausa) das mulheres entrevistadas, o que impossibilitou associar a sintomatologia ao estágio da menopausa no presente estudo. Além disso, as mulheres analfabetas foram excluídas do estudo devido à autoaplicação dos questionários.

Faz-se necessário destacar, também, que a prevalência da raça parda é um dado limitado, visto que tal característica pode ser atribuída ao fato do item ser autodeclarado, o que sofre influência de opiniões pessoais. Ademais, outro fator limitante é que na Escala de Cervantes não há um ponto de corte préestabelecido para classificação da qualidade de vida, logo, no presente estudo, como em muitos outros, essa classificação foi realizada através da média do escore total.

\section{CONCLUSÃO}

Foi observado a partir deste estudo que a síndrome climatérica interfere de forma negativa na vida das mulheres, uma vez que, quanto maior o impacto dos sintomas climatéricos menor é a qualidade de vida nas mulheres deste estudo e o inverso é verdadeiro. A partir desses dados, pode-se concluir que a atuação dos profissionais de saúde é essencial no que se diz respeito ao encorajamento de mudança de hábitos de vida e adoção de práticas saudáveis por essas mulheres, sobretudo do enfermeiro, o qual deve promover ações educacionais e orientações que fortaleçam uma maior autonomia e autocuidado nessa etapa da vida.

\section{REFERÊNCIAS}

1. ALVES ERP, et al. Climatério: a intensidade dos sintomas e o desempenho sexual. Texto contexto - enferm., Florianópolis, 2015; 24(1): 64-71.

2. ANDRADE RL, et al. Avaliação da qualidade de vida de mulheres climatéricas atendidas em ambulatório especializado. Brazilian Journal of Health Review. Curitiba, 2019; 2(1): 66-90.

3. ARAÚJO JBS, et al. Avaliação da Intensidade da Sintomatologia do Climatério em Mulheres: inquérito populacional na cidade de Maceió, Alagoas. Caderno de Graduação-Ciências Biológicas e da Saúde-FITS. 2015; 2(3), $101-111$.

4. BESSA AD, et al. O climatério segundo a percepção de mulheres em uma instituição privada de ensino superior do DF. In Simpósio de TCC e Seminário de IC. Anais do Simpósio I CESP Promove. 2016.

5. BRASIL. Ministério da Saúde. Secretaria de Vigilância em Saúde. Vigitel Brasil: vigilância de fatores de risco e proteção para doenças crônicas por inquérito telefônico. Brasília: MS; 2011.

6. BRASIL. Ministério da Saúde. Protocolos da Atenção Básica: Saúde das Mulheres / Ministério da Saúde, Instituto Sírio-Libanês de Ensino e Pesquisa - Brasília: Ministério da Saúde, 2016.

7. DEDICAÇÃO AC. Dor, qualidade de vida e depressão em mulheres climatéricas, adscritas a uma Unidade Básica de Saúde do município de São Paulo; São Carlos, 2012.

8. FREITAS ER, BARBOSA AJG. Qualidade de vida e bem-estar psicológico no climatério. Arq. bras. psicol., Rio de Janeiro, 2015; 67(3): 112-124. 
9. FREITAS ER, et al. Educação em saúde para mulheres no climatério: impactos na qualidade de vida. Reprodução e climatério. 2016; 31(1): 37- 43.

10. FREITAS RF, et al. Qualidade de vida de mulheres climatéricas em relação aos valores da circunferência abdominal. Geriatr Gerontol Aging. 2014; 8(4): 216-220.

11. FREITAS RF, et al. Qualidade de vida de mulheres climatéricas de acordo com o estado menopausal. Revista da Universidade Vale do Rio Verde. 2015; 13(1): 37-47.

12. FREITAS RF, et al. Qualidade de vida de mulheres climatéricas assistidas na Atenção Primária á Saúde. Revista Espacios. 2017; 38(36), 27-38.

13. GONÇALVES JTT, et al . Sobrepeso e obesidade e fatores associados ao climatério. Ciênc. saúde coletiva, Rio de Janeiro, 2016; 21(4): 1145-1156.

14. INSTITUTO BRASILEIRO DE GEOGRAFIA E ESTATÍSTICA (IBGE). Pesquisa de orçamentos familiares $2008-2009$. Antropometria e estado nutricional de crianças, adolescentes e adultos no Brasil. Rio de Janeiro; 2010.

15. LIMA CG, et al. Circunferência da cintura ou abdominal? Uma revisão crítica das referências metodológicas. Rev Simbio-Logias. 2011; 4(6): 108-31.

16. LIMA JEM. Tradução, Adaptação Cultural e Validação da Versão em Português Brasileiro da Escala Cervantes de Qualidade de Vida Relacionada com a Saúde da Mulher durante a Perimenopausa e na Pós-Menopausa. Universidade Federal do Rio Grande do Sul Faculdade de Medicina. RS, 2009.

17. LORENZI D, et al. Fatores associados à qualidade de vida após menopausa. Revista Da Associação Medica Brasileira - REV ASSOC MED BRAS. 2006.

18. MARTINS M, et al. Prevalência de disfunção sexual em mulheres climatéricas. Salão do Conhecimento, ago. 2015; ISSN 2318-2385.

19. MELO C, et al. Aplicação do Índice Menopausal de kupperman: um estudo transversal com mulheres climatéricas. Espaço para a Saúde - Revista de Saúde Pública do Paraná. 2016; 17(2): 41.

20. MIRANDA JS, et al. Qualidade de vida em mulheres no climatério atendidas na Atenção Primária. Rev Bras Enferm. 2014; 67(5): 803-9.

21. PEREIRA DCL, LIMA SMRR. Prevalência de sobrepeso e obesidade em mulheres após a menopausa. Arq Med Hosp Fac Cienc Med Santa Casa, São Paulo. 2015; 60: 1-6.

22. POLISSENI AF, et al. Depressão e ansiedade em mulheres climatéricas: fatores associados. Rev. Bras. Ginecol. Obstet., Rio de Janeiro, 2009; 31(1): 28-34.

23. RIBEIRO AS, et al. Avaliação dos sintomas e da qualidade de vida das mulheres no climatério. Revista da Universidade Vale do Rio Verde. 2015; 13(1): 48-65.

24. ROCHA ASG. O impacto dos sintomas climatéricos na qualidade de vida e função sexual. Centro Universitário Toledo. Araçatuba-SP, 2017.

25. RODRIGUES RM. Associação entre obesidade e sintomatologia climatérica. 2019. 25 f. Trabalho de Conclusão de Curso (Graduação em Educação Física) - Universidade Federal de Uberlândia, Uberlândia, 2019.

26. SANTOS JL, et al. Reprodução e climatério, 2016; 31:86-92- geral. 8,11-13.

27. SANTOS RCF, et al. Saúde de mulheres no climatério em sistema prisional. Cogitare Enfermagem, 2017; 22(1).

28. SILVA CB, et al. Atuação de enfermeiros na atenção às mulheres no climatério. Journal of Nursing UFPE on line, 2014; 9(1): 312-318.

29. SILVEIRA IL, et al. Prevalência de sintomas do climatério em mulheres dos meios rural e urbano no Rio Grande do Norte, Brasil. Revista Brasileira de Ginecologia e Obstetrícia, v.29 n.8. Rio de Janeiro-RJ, agosto, 2007.

30. THIEL RRC et al. Tradução para português, adaptação cultural e validação do Female Sexual Function Index. Rev. Bras. Ginecol. Obstet., Rio de Janeiro, 2008; 30(10): 504-510. 